\title{
Key paediatric messages from the 2018 European Respiratory Society International Congress
}

\author{
Raffaella Nenna ${ }^{10}{ }^{1,2}$, Katie A. Hunt ${ }^{3}$, Theodore Dassios ${ }^{3}$, Jennifer J.P. Collins ${ }^{4}$ \\ Robbert J. Rottier ${ }^{4}$, Norrice M. Liu ${ }^{5}$, Bart Rottier ${ }^{6}$, Myrofora Goutaki (1) 7 , \\ Bülent Karadag ${ }^{8}$, Andrew Prayle ${ }^{9}$, Ricardo M. Fernandes ${ }^{10,11}$, \\ Giuseppe Fabio Parisi $\mathbb{1}^{12}$, Jürg Barben ${ }^{13}$, Bruna Rubbo ${ }^{14,15}$, \\ Deborah Snijders ${ }^{16}$, Heidi Makrinioti ${ }^{17}$, Graham Hall $^{18}$, \\ Mariëlle W. Pijnenburg ${ }^{19}$ and Jonathan Grigg ${ }^{20}$
}

Affiliations: ${ }^{1}$ Dept of Paediatrics, Sapienza University of Rome, Rome, Italy. ${ }^{2}$ Asthma and Airway Disease Research Center, The University of Arizona, Tucson, AZ, USA. ${ }^{3}$ Women and Children's Health, School of Life Course Sciences, Faculty of Life Sciences and Medicine, King's College London, London, UK. "Dept of Paediatric Surgery, Sophia Children's Hospital, Erasmus University Medical Centre, Rotterdam, The Netherlands. ${ }^{5}$ Centre for Genomics and Child Health, Blizard Institute, Queen Mary University London, London, UK. ${ }^{6}$ Beatrix Children's Hospital, University Medical Centre Groningen, Groningen, The Netherlands. ${ }^{7}$ Institute of Social and Preventive Medicine, University of Bern, Bern, Switzerland. ${ }^{8}$ Division of Pediatric Pulmonology, Marmara University, School of Medicine, Istanbul, Turkey. ${ }^{9}$ Child Health, Obstetrics and Gynaecology, The University of Nottingham, Nottingham, UK. ${ }^{10}$ Dept of Pediatrics, Santa Maria Hospital, Lisbon, Portugal. ${ }^{11}$ Clinical Pharmacology Unit, Instituto de Medicina Molecular, Faculty of Medicine, University of Lisbon, Lisbon, Portugal. ${ }^{12}$ Dept of Clinical and Experimental Medicine, University of Catania, Catania, Italy. ${ }^{13}$ Division of Paediatric Pulmonology, Children's Hospitals of Eastern Switzerland, St. Gallen, Switzerland. ${ }^{14}$ Primary Ciliary Dyskinesia Centre, NIHR Biomedical Research Centre, University Hospital Southampton NHS Foundation Trust, Southampton, UK. ${ }^{15}$ University of Southampton, Faculty of Medicine, Academic Unit of Clinical and Experimental Science, Southampton, UK. ${ }^{16}$ Dept of Women's and Children Health, University of Padova, Padova, Italy. ${ }^{17}$ Queen Mary University London, London, UK. ${ }^{18}$ Telethon Kids Institute and Curtin University, Perth, Australia. ${ }^{19}$ Dept of Paediatrics, Division of Paediatric Pulmonology and Allergology, Erasmus MC-Sophia Children's Hospital, University Medical Centre Rotterdam, Rotterdam, The Netherlands. ${ }^{20}$ Centre for Paediatrics, Queen Mary University London, London, UK.

Correspondence: Raffaella Nenna, Dept of Paediatrics, "Sapienza" University of Rome, V.le Regina Elena 324 00161, Rome, Italy. E-mail: raffaella.nennaduniroma1.it

ABSTRACT In this article, the Group Chairs and early career members of the European Respiratory Society (ERS) Paediatric Assembly highlight some of the most interesting findings in the field of paediatrics which were presented at the 2018 international ERS Congress.

@ERSpublications

The 2018 international \#ERSCongress in Paris, France reflected the @ERSTalk's commitment to support the continuing professional and academic development of the 1500 members of the Paediatric Assembly. http://ow.ly/P2Ck50pDRy6

Cite this article as: Nenna R, Hunt KA, Dassios T, et al. Key paediatric messages from the 2018 European Respiratory Society International Congress. ERJ Open Res 2019; 5: 00241-2018 [https://doi.org/10.1183/23120541.00241-2018]. 


\section{Introduction}

The 2018 international European Respiratory Society (ERS) Congress in Paris, France reflected the ERS's commitment to support the continuing professional and academic development of the 1500 members of the Paediatric Assembly (Assembly 7). Members of the Assembly presented over 350 scientific abstracts in addition to a postgraduate course on cystic fibrosis (CF), two meet the expert sessions (on bronchoscopic evaluation of recurrent pneumonia and primary ciliary dyskinesia (PCD)), a skills workshop on endoscopy, four scientific symposia, one year in review session, the paediatric grand round and a state-of-the-art session. A new, and very successful, format was the "lung on fire" session, where a panel of paediatric experts were put to the test by clinical cases provided by the audience. In previous years, the senior officers of the Assembly have reviewed the highlights of the Congress $[1,2]$. In contrast, this year's review of the Congress was a partnership between our Early Career Members (i.e. members aged $<40$ years) and the Assembly Group Chairs. Each pair report what they personally found to be the most interesting findings of the Congress, inspired by data presented in both abstracts and spoken sessions.

\section{Prematurity and lung disease}

New insights into prematurity and lung disease ranged from interventions following premature delivery to optimise lung aeration, through early biomarkers and predictors of future chronic lung disease, to the later pulmonary consequences of prematurity.

Bizzoтtо et al. [3] randomised lambs delivered prematurely at 128 days (approximately equivalent to 28 weeks gestation in humans) to receive two sustained inflations or continuous positive airway pressure immediately after delivery. All lambs were subsequently maintained on noninvasive positive pressure ventilation. Forced oscillation at $5 \mathrm{~Hz}$ was used to assess lung aeration. Whilst the lambs that received sustained inflations had greater and more homogenous lung aeration, differences were only significant for the first 3 hours of life. This may help to explain why although sustained inflations may reduce the need for ventilation, benefits do not appear to be sustained in the long term $[4,5]$.

Another animal study of the effects of preterm birth by DAHL et al. [6], addressed the long-term respiratory consequences of preterm birth. In this model, lambs were ventilated for 3-6 days and then weaned from support. Compared to control lambs, airway reactance was increased in the first 2 months of life. At the equivalent age of $\sim 6$ years in humans, the preterm lambs had significantly increased airway smooth muscle thickness compared to controls, despite normal reactance and lung mechanics at that time.

If oxidative stress contributes to the development of bronchopulmonary dysplasia (BPD), then antioxidant gene variants may influence susceptibility [7]. PAvLINova et al. [8] investigated allelic polymorphisms of manganese superoxide dismutase (MnSOD) by isolating genomic DNA from infants with and without BPD. The researchers found that substitution of cytosine for thymine at position 16 on the MnSOD gene (resulting in substitution of valine for alanine) was present in $90 \%$ of infants with BPD compared to only $32 \%$ without BPD ( $p=0.006$ ), whereas two other mutations showed no such increased susceptibility. This underlines the hypothesis that antioxidant gene variants are involved in the multifactorial aetiology of BPD.

Two abstracts addressed methods to predict subsequent development of BPD in "at risk" infants. ZANNIN et al. [9] used forced oscillation in 68 non-intubated infants born at $<32$ weeks. Respiratory system reactance $(X \mathrm{rs})$ was associated with duration of respiratory support, and the best multivariate model predicting $\mathrm{BPD}$ incorporated gestational age and $X_{\mathrm{rs}}(\beta=-0.62,95 \% \mathrm{CI}-1.07--0.17 ; \mathrm{p}=0.008)$. A late-breaking abstract from KINDT-DUNJKO et al. [10] used machine learning to identify different protein signatures that might predict BPD development. In this study, plasma samples were taken at day 1-7 from infants who went on to develop mild BPD compared to those who developed moderate or severe BPD. Proteins implicated in disease severity of later BPD were those involved in inflammatory signalling, cardiac strain and coagulation activation.

One goal of early identification of infants at risk of a higher burden of respiratory disease is to target potential future therapies. A potential therapeutic target was reported by Gallacher et al. [11] who showed that extracellular apoptosis-associated speck-like protein containing a caspase activation domain was instrumental in interleukin (IL)- 6 and IL-8 production from respiratory cell analogues, and that this may represent a potential therapeutic target to prevent chronic lung disease.

There is evidence to suggest that azithromycin has the potential to reduce the development of BPD, but this approach may only have a significant benefit in infants colonised with ureaplasma [12, 13]. VISCARDI et al. [14] presented the results of a double-blind placebo-controlled trial of intravenous azithromycin $\left(20 \mathrm{mg} \cdot \mathrm{kg}^{-1}\right)$ in the prevention of severe respiratory morbidity in infants born at $24-28+6$ weeks. Infants with tracheal aspirates positive for ureaplasma had higher mortality $(29 \%$ versus $10 \%, \mathrm{p}<0.01)$, and higher rates of $\mathrm{BPD}(67 \%$ versus $50 \%, \mathrm{p}<0.01)$. By contrast, tracheal aspirate ureaplasma positive infants that 
received azithromycin had lower rates of death or severe respiratory morbidity at 1 year than the placebo group (three (33\%) out of nine versus six (86\%) out of seven, $\mathrm{p}=0.036)$.

Even longer term outcomes of prematurity were addressed by HARRIs et al. [15] who found that self-reported exercise (measured as how much time per week children exercised) correlated poorly with performance on exercise testing in adolescents who had been born prematurely. Poorer lung clearance index (LCI; a measure of ventilatory inhomogeneity) was predictive of poorer performance, but other lung function tests were not correlated with exercise distance, suggesting that lung function abnormalities may not always be associated with functional impairment.

\section{Molecular and cellular determinants of the developing lung}

The thematic poster session "Molecular and cellular determinants of the developing lung" was the first official ERS session for the new Group 7.08: Lung and Airway Developmental Biology, which was formed in 2018. This session featured 12 posters covering the newest studies on normal lung development, hyperoxia-induced neonatal lung injury, asthma/airway hyperreactivity and developmental origins of chronic lung disease.

Using single cell RNA sequencing on human fetal lungs, DANOPOUlos et al. [16] showed that various distinct mesenchymal lineages, including endothelial cells, pericytes, smooth muscle cells and fibroblasts, are already specified in pseudoglandular (11.5 weeks) lungs. A study by WANG et al. [17] focused on the maturation of the airway smooth muscle (ASM) layer during late gestation to the first year in life in human lungs. Using stereological techniques, they found that the ASM layer thickens with a concomitant reduction in ASM cell density as a result of hypertrophy. In turn, MiURA et al. [18] presented data implicating a role for epithelial phosphatase and tensin homolog deleted on chromosome 10 in controlling programmed cell senescence and cell fate choices during lung development in mice, by regulating the NF- $\mathrm{B}-$ Notch pathway.

Studies aiming at elucidating processes involved in hyperoxia-induced injury of the newborn lung, as a model for BPD in preterm infants, were particularly well represented in this session. In a study by CoLLins et al. [19], which was selected from the 2018 Lung Science Conference in Estoril, Portugal, CD146+ lung mesenchymal stromal cells (L-MSCs) isolated from hyperoxia-injured rat lungs displayed an impaired angiogenic supportive capacity and altered gene expression profile, suggesting an active role for L-MSCs in BPD pathogenesis [20]. Hirani et al. [21] presented data suggesting an injurious effect of macrophages on lung epithelial cells following hyperoxia exposure, mediated by IL-6. Loss of IL- 6 on the other hand protected against hyperoxia-injury in neonatal mice. HIRANI et al. [22] also presented data on the downregulation of Krüppel-like factor 4 (Klf4) in myofibroblasts following hyperoxia exposure in neonatal mice, linking this to myofibroblast activation and potential contribution to BPD pathogenesis. MoHR et al. [23] also identified a role for Klf4 in alveolar epithelial type II cells, which was diminished after hyperoxia exposure in neonatal mice. Potential therapeutic targets for the treatment of BPD were also presented. SYED et al. [24] showed that an agonistic antibody aimed at the triggering receptor expressed on myeloid cells-1, which is upregulated as an adaptive response to hyperoxia in neonatal mouse and human lungs, could alleviate pulmonary alveolar injury and inflammation by downregulating receptor-interacting protein kinase 3-mediated cell death. Using a hyperoxia-induced newborn guinea pig model of experimental BPD, KATOVICH et al. [25] reported that inhalation therapy with $\mathrm{N}$-acetylcysteine could prevent alveolar hypoplasia through normalisation of the redox balance and an improved elastase/A1-AT ratio.

Exposures to the developing lung can not only lead to effects that are directly apparent, but can also predispose for chronic lung disease that manifests at a later age. The usefulness of zebrafish as a potential novel animal model for chronic lung disease in this regard was presented in a study by LjujicH et al. [26], showing a correlation between the effects of nicotine exposure on mammals and zebrafish. In turn, PINCUs et al. [27] looked at maternal house dust mite exposure in mice as a model for asthma, finding that this increases sensory airway innervation and airway hyperreactivity in offspring. Lastly, presenting a late breaking abstract, KovacH et al. [28] obtained serum from dog-allergen sensitised children, and found that while IL-26 and IL-17A concentrations were increased in this group compared to those without allergen sensitisation, IL-26 also correlated with improved asthma control. This would support the view that exposure to pets indeed has a protective effect in the early origins of asthma.

\section{Paediatric asthma: prevention, diagnosis and treatment}

Presentations at the ERS Congress covered the wide spectrum of asthma, including prevention, diagnosis and treatment. Exploring the intriguing association between pre-natal maternal psychological stress [29], LIU et al. [30] performed a population-based study on Danish children born between 1997 and 2008, examining the relationship between maternal pre-natal stress and the risk of offspring asthma. Whilst private life stress (e.g. bereavement and illness) did not influence the risk, maternal exposure to lower job demand and with low job control increased the risk. 
Another emerging protective factor for asthma is the interaction between exposure to bacterial products and microbiota. One source of early life microbial exposure is via breast milk. HämYNEN et al. [31] explored the connection between microbiota in breast milk and asthma by 6 years of age. Breast milk samples were collected from mothers from the PASTURE birth cohort (Finland, France, Germany and Switzerland). Differences in bacterial strains were found in different countries; gram-negative bacteria showed a significant association with asthma in Germany and Switzerland. The respired environment is another source of bacterial product exposure. Previous reports that asthma is less prevalent in children exposed to a wider range of microbes (e.g. living on farms) [32] suggests a role for either inhalation or ingestion of bacterial products. PEKKANEN et al. [33] studied the differences in house dust microbiota composition between farm and non-farm homes in Finland. Floor dust bacteria and fungus were sequenced at the age of 2 months, while the outcome was defined as asthma by 6 years of age. Farm home-like microbiota showed a protective effect, as seen in the GABRIELA study [32]. The researchers concluded that the composition patterns were more specific than bacteria population richness.

Preschool wheeze, which affects one in three children before the age of 3 years, remains a conundrum [34]. It remains unclear whether preschool children whose wheeze transitions in to school age asthma have the same pathological entity, or are at risk of developing a separate pathological condition. Even in asthma in school age children, diagnostic uncertainty is common suggesting a marked degree of heterogeneity in underlying pathology [35]. Typical diagnostic tests include spirometry, exhaled nitric oxide (FeNO) and atopy investigations. FeNO is a biomarker of airway inflammation which can be useful in the diagnosis and monitoring of asthma. However, there is limited longitudinal data on FeNO trajectories. EcKel et al. [36] proposed that serial measurements of FeNO may have clinical applications. They analysed a cohort of non-asthmatic children from the Southern California Children's Health Study. Baseline FeNO had the highest importance in determining FeNO trajectories. Whilst trajectories for all children are heterogeneous, healthy children tend to follow their own course throughout childhood. The researchers confirmed FeNO increases with age and height in healthy children, and that serial FeNO measurements have clinical uses. Another set of compounds in breath that may provide new insights are volatile organic compounds (VOCs) [37]. The "electronic nose" (eNose) uses exhaled VOCs to identify diseases [38], but studies on its use in children are limited. BANNIER et al. [39] examined the feasibility and diagnostic accuracy of eNose in children with asthma, CF and in healthy controls. 55 participants aged 5-20 years underwent eNose breath sampling. With $98 \%$ successful measurements, the device was easy to use, provided high accuracy in discriminating CF from asthma and healthy controls, and modest accuracy in distinguishing asthma from healthy controls.

Whilst asthma treatments for mild-to-moderate asthma are well established, delivery of drug to the lower airway and adherence in children remain problematic. Valve holding chambers (VHCs) enhance drug delivery and eliminate coordination of actuation and inhalation. Commercially available VHCs have different volumes, aerodynamics and mechanisms, but they are used interchangeably, assuming equal efficacy. Csonka et al. [40] assessed drug delivery with various VHCs while simulating different breathing patterns (combinations of respiratory rate and tidal volume). There were marked dose delivery differences between VHCs, though the differences remain constant for different breathing patterns. These researchers stressed the importance of developing guidelines on what inhaler/VHC combination to use for what age group.

In severe asthma, oxygen therapy is often used, but administration methods vary widely (e.g. temperature and humidity). СомртоN et al. [41] compared the outcomes of children randomised to standard oxygen, humidified oxygen or warmed humidified oxygen. Surprisingly, warmed humidified oxygen was poorly tolerated and associated with treatment escalation. Taking into account these were pilot data, the authors encouraged larger studies to standardise practice.

\section{Insights into respiratory disease from epidemiological studies}

Several studies using epidemiological approaches in clinical and population-based research were presented at the Congress, focusing mostly on asthma and childhood wheeze but also on other paediatric respiratory disorders such as primary ciliary dyskinesia. Research on pre-natal and post-natal early life factors and their influence on later respiratory disease was the main theme of an oral session on "Recent insights in childhood asthma and wheezing". Pre-natal paracetamol exposure has been positively associated with asthma development but not all studies have been consistent [42, 43]. BREw et al. [44] investigated the association between analgesic use in pregnancy and childhood asthma by using family design methods to assess possible confounding. Studies using sibling comparisons, where the mother took analgesic in one pregnancy and not in the other, allow to control for genetic factors, extrinsic confounding and partially for intrinsic confounding. The study population included 492999 mother-child pairs identified from Swedish national registries. Analgesic use during pregnancy was identified from prescription data for paracetamol, 
anti-migraine drugs and codeine. Childhood asthma was assessed at ages 2, 3 and 4 years based on asthma diagnosis and use of asthma medication [45]. All pre-natal analgesic use was associated with increased risk of childhood asthma at all ages; however, the siblings comparison found no association between pre-natal analgesic use and childhood asthma, suggesting that there might be possible confounding related to the maternal factors. DAI et al. [46] investigated another aspect of analgesic use, studying the association between paracetamol during infancy and risk of asthma in adolescents using data from the Method-Melbourne Atopy Cohort Study [47]. After adjusting for frequency of early respiratory infections, paracetamol use during the first 2 years of life was not associated with wheeze or lower lung function at 18 years of age. However, the study found an increased risk for lower lung function in adolescents with GSTM1 null genotypes and GSTP1 Ile/Ile genotypes indicating that extensive use of paracetamol in children susceptible to oxidative stress might be linked to long-term respiratory outcomes. Котесна et al. [48] presented data from the Millennium Cohort Study [49] to identify wheezing phenotypes using machine learning to estimate different latent class models. Recent wheeze was greater in the preterm-born group at ages 3,5, 7 and 11 years and four wheezing phenotypes were found, which were similar for both groups: 1) no/infrequent wheeze; 2) early wheeze which disappeared in later years; 3 ) persistent wheeze throughout the study period; and 4) late wheeze which appeared later in childhood. Preterm infants were more likely to have early or persistent wheeze. The study showed similar early life risk factors for both preterm and term-born children (e.g. atopy, antenatal maternal smoking, no breastfeeding and male sex) but their effect was stronger in preterm children. CASAS et al. [50] focused on early growth patterns which have been implicated to childhood asthma and lung function [51, 52], using repeated growth measurements for the first 3 years of life of 4435 children from the Generation R study [53]. They used detailed individual growth trajectories to assess the association of peak height and weight growth velocity, body mass index and age at adiposity peak with lung function and reported asthma at age 10 years [50]. Greater peak weight velocity and greater body mass index at adiposity peak were associated with a disproportionate increase in forced vital capacity (FVC) compared to forced expiratory volume in $1 \mathrm{~s}(\mathrm{FEV} 1)$, leading to smaller airways in relation to lung capacity. Animal and in vitro studies showed an effect of vitamin D on lung and immune system development but there is little data from human studies [54, 55]. Using data from the Generation $\mathrm{R}$ study, Mensink-Bout et al. [56] examined the association of 25-hydroxyvitamin D (25(OH)D) blood concentration in mid-gestation and at birth with asthma and allergy outcomes at age 10 years. They found that higher levels of $25(\mathrm{OH}) \mathrm{D}$ mid gestation were associated with a higher risk of inhalant allergen sensitisation at school age, partially explained by the child's $25(\mathrm{OH}) \mathrm{D}$ levels. All studies highlighted the importance of cohort studies and the use of appropriate methods to clarify possible associations of early life factors with respiratory outcomes in childhood.

\section{Paediatric respiratory infections: translational and clinical perspectives}

Several of the Congress sessions addressed paediatric respiratory infections, and excellent abstracts were presented in this area encompassing epidemiological, translational and clinical research studies. AN et al. [57] assessed whether induced sputum was an alternative to bronchial fluid to investigate the lower airway microbiota. In a small sample of 12 children undergoing elective ear, nose and throat procedures (six with known asthma), the microbiota in induced sputum and bronchial samples were found to be different, with the former closer to that of upper airway samples. The authors also found that a minority of bacterial DNA in bronchial samples remained unclassified. These findings suggest that induced sputum is not a valid surrogate for direct bronchial sampling to study the lower airway microbiota in all children.

$\mathrm{PoH}$ et al. [58] reported on the interaction between non-typeable Haemophilus influenzae (NTHi) and respiratory syncytial virus (RSV) in a model of primary airway epithelial cells growing at the air-liquid interface. RSV infection enhanced the growth of NTHi, but the opposite was not the case. NTHi produced a confluent biofilm over the whole epithelial surface of the culture well, likely preventing contact of RSV with epithelial cells. In vivo, biofilms are not likely to form this barrier throughout the bronchial surface; modifications to this experimental model may be required to further study the interaction between RSV and NTHi.

The roles of RSV genotype and host-immune interactions were presented by PIERANGELi et al. [59], using clinical and biomarker data from a single-centre study of 96 inpatient infants with bronchiolitis. The authors measured viral load and interferon-stimulated genes (MxA and ISG56) in nasopharyngeal aspirates across two time-periods. The NA1 genotype predominated between 2005 and 2012, followed by the ON1 genotype until 2017. Reduced severity of illness across a range of clinical outcomes was found during the latter period. The NA1 genotype was associated with a lower viral load, but increased expression of MxA and ISG56. These results provide further mechanistic insight on host-agent interactions in RSV bronchiolitis, which are particularly relevant given the pipeline of new antivirals currently in development. 
Two abstracts presented data on the association of infant bronchiolitis and lung function measurements at later age. CANGiano et al. [60] assessed ventilation inhomogeneity using multiple breath washout measurements at 5-6 years of age in a cross-sectional study. Children with a history of bronchiolitis $(n=29)$ had a higher LCI than age-matched healthy controls $(n=15)$, but less than children with CF $(n=20)$. RiIKONEN et al. [61] reported on follow-up spirometry at 10-13 years of age from a matched cohort study comparing children hospitalised with bronchiolitis before 6 months of age $(n=89)$ and controls $(\mathrm{n}=108)$. This group had previously reported reversible obstruction (with forced oscillometry) at age 5-7 years in this cohort [62]. Early-life bronchiolitis was associated with reduced FEV1 and FEV1/FVC before and after bronchodilation, including after adjustment for maternal smoking and current asthma. Findings from these studies suggest that changes in lung function after early-life bronchiolitis are seen at preschool age, and there may be irreversible bronchial obstruction by early adolescence. Methodological caveats in establishing the causal effect of bronchiolitis include the selection of controls and the fact that pre-existing factors such as lung function at birth were unaccounted for.

UNGER et al. [63] aimed to address a gap in evidence regarding the role of capillary blood gas analysis in management of bronchiolitis. In a single-centre retrospective observational study over two bronchiolitis seasons, blood gas was performed in 158 out of 326 hospitalised infants, 98 in the emergency department. Elevated carbon dioxide tension was associated with high-dependency or intensive care unit admission (OR 2.8; 95\% CI 1.2-6.6) and length of hospital stay. Future studies designed to reduce biases inherent to this study design would help further elucidate the prognostic value of blood gas, which would be relevant for both clinical practice and future intervention trials.

Finally, RufFles et al. [64] presented the 5-year outcomes of a large cohort of children with protracted bacterial bronchitis that underwent bronchoscopy $(n=130)$. A considerable proportion of children who underwent computed tomography had bronchiectasis (11 out of 59) and 15.7\% had recurrent disease (>3 episodes per year) by year 5 . Interestingly, $45.9 \%$ had a diagnosis of asthma. While there may be limits to the extrapolation of these results, identifying prognostic markers of future airways disease at the index of protracted bacterial bronchitis episode would be useful.

\section{CF: diagnosis, monitoring, microbiological aspects and new treatments}

The ERS Congress addressed the significant advances that have been made in CF management in the past decade [65].

The oral communication session focused on microbiological aspects and new treatments in CF. LiNNANE et al. [66] described a prospective case-control study on the lower airway microbiome in pre-school children with CF. Data on 291 sequenced bronchoalveolar lavages from 50 controls and 106 CF patients showed that CF lower airway microbiome is distinct from the non-CF microbiome, had lower diversity, and different relative abundance at phylum, family and genus level.

BRINKAm et al. [67] studied the role of exhaled breath in the detection of Pseudomonas aeruginosa of CF patients. They found that three out of 138 VOCs in the exhaled breath were consistently different in $P$. aeruginosa infected versus non-infected patients.

This pilot study provided support for the use of exhaled VOC analysis for early detection of P. aeruginosa infections in CF patients and therefore help with prompt treatment.

Another study on volatile metabolites in the breath by BAGHDASARYAN et al. [68] presented on distinct volatile markers from CF pathogens with secondary electrospray ionisation high-resolution mass spectrometry (SESI-HRMS). With this technique, unique sets of four to 12 volatile markers were found for P. aeruginosa, H. influenzae, Streptococcus pneumoniae, Staphylococcus aureus and Escherichia coli. Several markers for P. aeruginosa, Burkholdia cepacia and Stenotrophomonas maltophilia were closely related.

STAHL et al. [69] presented the randomised, double-bind, controlled trial of preventive inhalation of hypertonic saline in infants with CF (PRESIS) study. The aim of that study was to determine safety and efficacy of preventive inhalation of $6 \%$ hypertonic saline versus $0.9 \%$ isotonic saline in 42 infants with CF. They demonstrated that inhalation with hypertonic saline is safe in infants, with good adherence and it is associated with a reduction in the LCI and a significant better body weight gain after 12 months when compared to isotonic saline alone.

Bush et al. [70] discussed targeting $\alpha$-epithelial sodium channel (ENaC) with an epithelial RNAi trigger delivery platform for the treatment of CF. This complex work aimed to evaluate the efficacy of the Arrowhead Targeted RNAi Molecule technology to develop epithelial-targeted conjugates comprised of an optimised RNAi trigger against $\mathrm{ENaC}$ paired with an epithelial targeting ligands to the epithelial integrin $\alpha v \beta 6$. They demonstrated that inhaled epithelial targeting ligands $-\alpha E N a C$ RNAi trigger conjugates 
produce selective, durable, renal-sparing silencing of pulmonary $\alpha \mathrm{ENaC}$ expression. Moreover, improved mucociliary clearance was observed in sheep 2 weeks after inhalation of aerosolised conjugate.

The second oral communication session focused on the new developments in diagnosis and monitoring in CF.

JENSEN et al. [71] reported the effects of deviations in tidal breathing on multiple breath washout measurements. Using a large retrospective dataset, the authors demonstrated that within the range of tidal volumes observed, minor deviations from ideal tidal volume and sighs did not impact end-tidal nitrogen concentration, and had only small effects on functional residual capacity and LCI that are unlikely to be clinically relevant.

CIUCA et al. [72] evaluated the relationship between lung ultrasound score and lung function expressed by LCI in a cohort of 42 children with CF. The authors found an important and statistically significant correlation between lung damage evaluated by the lung ultrasound score and the LCI. Thus, they speculated that lung ultrasound score might be used as a primary evaluation of CF lung damage.

NYILAS et al. [73] presented on the repeatability of ventilation and perfusion assessment by functional magnetic resonance imaging (MRI) in children with CF. Pulmonary MRI techniques such as matrix pencil decomposition allow for the evaluation of regional impairment of the regional fractional ventilation and relative perfusion in patients with $\mathrm{CF}$. However, the repeatability of matrix pencil-MRI outcomes in children with CF is unknown. The authors demonstrated a good short-term repeatability, making this technique promising for early detection of CF lung disease.

SMITH et al. [74] expanded the MRI theme further in their presentation on the effect of inhaled lung volume on ventilation heterogeneity in CF using $3 \mathrm{He}$ MRI. In patients with $\mathrm{CF}$, ventilation abnormalities using hyperpolarised gas ventilation MRI are a very early sign of disease (more sensitive than LCI or spirometry) but the relationship between tests of lung function and ventilation MRI has only been assessed in mild CF disease. The authors demonstrated that there is a strong correlation between $3 \mathrm{He}$ MRI and the LCI. Moreover, deep inspiration reduces, but does not always cause, ventilation abnormalities to disappear. This has implications for scanning techniques.

HARDY et al. [75] presented the comparison between the 1-min sit-to-stand test and quadriceps strength in patients with CF. The 1-min sit-to-stand test has the capacity to assess peripheral muscular strength and endurance and has not been well evaluated in CF. The authors demonstrated that the 1-min sit-to-stand test could be a good alternative to the dynamometer to estimate quadriceps strength in stable patients with CF. Moreover, this test is easily realisable during routine visits and reproducible.

Finally, Frost et al. [76] discussed their work on the association between glycaemic variability indices and pulmonary function decline. This was a retrospective analysis of 56 CF patients undergoing continuous glucose monitoring. All measures of glycaemic variability showed inverse correlations with baseline FEV1, though there was no clearly superior outcome measure of the different methods presented.

All presentations provoked interesting discussion from the audience, and show how research in the field of $\mathrm{CF}$ is extremely lively and at the cutting edge of a number of different techniques.

\section{Recent advances in PCD}

PCD is a rare hereditary disorder, with disease-causative mutations reported in $>39$ genes to date and a prevalence of approximately 1:10000 [77, 78].

Clinical predictive tools have been developed to guide referrals for diagnostic testing $[79,80]$. Nasal nitric oxide (nNO) is low in patients with PCD but can be normal in patients with specific mutations [81, 82]. Additionally, MARTHIN et al. [83] have recently shown that nasal nitric oxide decreases during respiratory tract infections. The ultrastructure of motile cilia, which can be examined by transmission electron microscopy, consists of nine microtubule doublets and central pair. The nodal cilia at the embryonic node lack the central pair, resulting in a rotational movement that determines the left-right pattern of major organs. Disruption of this flow can lead to situs inversus totalis in $\sim 50 \%$ of patients, with complete mirror image of major organs or various combinations of laterality defects, the latter can be associated with congenital heart disease. Ciliary biopsy for evaluation of cilia function through high-speed video microscopy can show abnormal beat pattern; however, subtle abnormalities can be difficult to spot and secondary defects due to infection are common [84].

Mutations in DNAH5 account for a $25 \%$ of all patients. Recent studies have expanded the understanding of PCD genetics, with the discovery of genes that encode proteins located in the cytoplasm (i.e. pre-assembly genes) [85] and mutations on X-linked genes (e.g. PIH1D3), where a single heterozygous mutation can be disease-causative in men [86]. DAVIs et al. [87] reported worse respiratory phenotype in 
patients with CCDC39 and CCDC40 and less severe phenotypes were described in some patients with RSPH1 and CCDC103 mutations, highlighting the fact that PCD is a heterogeneous disease [77].

However, despite significant advances in PCD genetics, it remains a complex field, with approximately $25 \%$ of PCD patients not having an identified bi-allelic mutation in a known gene. New diagnostic modalities include electron tomography and immunofluorescence. The ERS diagnostic guidelines and the recently published American Thoracic Society guidelines provide important guidance on diagnostic testing for PCD but there are significant differences between the two, reflecting different target audiences and expertise [88, 89]. Hallmark transmission electron microscopy defects and bi-allelic (or X-linked) mutations on a known PCD-causative gene confirms the diagnosis of PCD; however, combination testing remains essential to exclude the diagnosis.

Clinical symptoms reported by patients vary with age [78]. Similarly, pathogen prevalence varies with age and colonisation by $P$. aeruginosa associated with lower lung function [90]. Importantly, a recent international study showed that lung function is similarly impaired in patients with PCD and CF [91].

The sperm flagella and the fimbriae of fallopian tubes exhibit a similar structure to respiratory cilia, leading to fertility problems in men and women. Recently, a retrospective study with 85 PCD patients reported over half of the women had children, 39\% through spontaneous conception and 17\% after assisted fertility. Spontaneous conception was lower in men (24.5\%) [92].

PCD management is currently based on two expert consensus reports [93, 94], largely extrapolated from CF and non-CF bronchiectasis management as there are very few longitudinal studies in PCD. PAFF et al. [95] reported no difference in quality of life between patients on hypertonic saline and isotonic saline in a double-blind crossover randomised controlled trial. However, it was underpowered and the primary outcome was not disease specific. Another randomised control trial is investigating the efficacy and safety of azithromycin prophylaxis [96] and a crossover randomised controlled trial is evaluating the safety and efficacy of VX-371 inhalation, an ENaC inhibitor. Smoking cessation, immunisation, physical activity, airway clearance and antibiotics to treat exacerbations and eradication of $P$. aeruginosa are currently indicated for the management of PCD patients, with other therapies such as hypertonic saline, azithromycin prophylaxis and bronchodilators used on a case-by-case basis.

The breadth and depth of PCD research at the 2018 ERS Congress highlighted the need for international collaboration to advance PCD research. Initiatives such as the international PCD Registry, the North American PCD Registry and the iPCD cohort represent an important step towards standardised collection of clinical and diagnostic data for this rare disease [78].

\section{New ideas about respiratory and sleep physiology in children}

WEBER et al. [97] presented their work around validation of the Sleep Clinical Record Protocol which was created by an Italian group. The tool was tested in 51 Brazilian children with obstructive sleep disorders and tonsillar hypertrophy. There was no difficulty in the use of the tool in different groups of patients. The sensitivity was 0.89 and specificity was 0.19 , compared to polysomnography data. The quite low specificity could be associated with the sample selection bias (tested mainly in patients requiring adenotonsillectomy). Validation of the tool in a more representative group of patients will follow.

Short lingual frenulum at birth has been associated with increased obstructive sleep apnoea in school age years. This observation was confirmed in a retrospective study of 547 children of mean age 9.6 years presented by EVANGELISTI et al. [98]. 140 out of 547 children with obstructive sleep apnoea had short frenulum and, in these children, lower tongue strength was measured.

No studies have specifically clarified the need for carbon dioxide monitoring in paediatric sleep disordered breathing assessment. TRUCCIO et al. [99] attempted to determine how often overnight carbon dioxide monitoring could change management of patients when added to cardiorespiratory polygraphy data. This was a retrospective study of 2 years using data on paediatric patients referred for investigation of sleep disturbed breathing. In 107 (82\%) out of 130 otherwise healthy children, obstructive sleep apnoea was diagnosed. Carbon dioxide did not change management when added to cardiorespiratory polygraphy. However, in 20 out of 383 children with pre-existing medical conditions there were detectable carbon dioxide changes that guided management. Therefore, overnight carbon dioxide monitoring was not proven to be necessary for the diagnosis and management of obstructive sleep apnoea in otherwise healthy children but was crucial for the management of patients with comorbidities.

A population survey study in the UK, analysed by a Scottish team [100], looked at best management approaches in patients with Robin sequence. From analysis of 153 cases, evenly spread across the UK, it was shown that a non-surgical approach in the management of upper airway obstruction, involving use of nasopharyngeal airways, can be equally effective compared to a surgical approach. 
Although common thinking would associate positively the in utero tobacco exposure with lung deficits, there are controversial data deriving from birth cohort studies. Glutathione s-transferase is an enzyme involved in detoxification and its active genotype has been associated with better lung function in infants. OwENs et al. [101] from Australia presented their birth cohort study relating lung function in early life with in utero triggers, more specifically tobacco exposure. They identified that risk for development of significant lung deficits is higher in infants with maternal tobacco exposure lacking the active genotype of this enzyme.

Practice varies regarding assessment of bronchodilator response in children with normal baseline spirometry and this is an important question to answer since nearly one-third of asthmatic children have normal baseline spirometry. BEYDON et al. [102] conducted a retrospective study showing that children with negative bronchodilator responses are often prescribed inhaled steroids and that normal baseline spirometry but abnormal main airway resistance is correlated with positive bronchodilator responses. Therefore, basing diagnosis and management on baseline spirometry needs a revisit.

VerhegGen et al. $[9,103]$ from Australia presented their work around validation of the hypoxia challenge test in preterm infants. The tool was validated in 30 preterm and 24 term, predominantly male, infants taking in total 54 domestic flights in Australia lasting from 1.1 to $5.5 \mathrm{~h}$. Parents were holding portable oxymeters and were recording behaviour (including sleep or awake). The test failed to predict oxygen requirement in flight. Factors associated with both the infant (variation between flights) and physiological changes (peripheral oxygenation, changes in altitude) could potentially explain this outcome.

As discussed above, predictive markers for short- and long-term respiratory morbidity in premature infants would be clinically useful. In the area of respiratory physiology, Usemann et al. [104] from Switzerland reported data on whether intraindividual variability of ventilation inhomogeneity and capnographic parameters is associated with subsequent respiratory morbidity and could potentially enhance the predictive power of BPD severity classification. Using a prospective birth cohort study of 133 preterm infants the researchers found that preterm infants who require rehospitalisation due to respiratory complications during the first year of life have a lower variability of several tidal breathing parameters shortly after birth and the BPD severity score had very low predictive power while by adding tidal volume or carbon dioxide variability to the predictive model, there were small improvement in performance.

Conflict of interest: R. Nenna has nothing to disclose. K.A. Hunt reports personal fees from Chiesi and Medimmune, and grants from SLE Ltd, outside the submitted work. T. Dassios has nothing to disclose. J.J.P. Collins has nothing to disclose. R.J. Rottier has nothing to disclose. N.M. Liu has nothing to disclose. B. Rottier has nothing to disclose. M. Goutaki has nothing to disclose. B. Karadag has nothing to disclose. A. Prayle has nothing to disclose. R.M. Fernandes has served on independent data monitoring committees for studies sponsored by Ablynx, and participated in clinical trials sponsored by Janssen. G.F. Parisi has nothing to disclose. J. Barben has nothing to disclose. B. Rubbo has nothing to disclose. D. Snijders has nothing to disclose. H. Makrinoti has nothing to disclose. G. Hall has nothing to disclose. M.W. Pijnenburg has nothing to disclose. J. Grigg reports personal fees from GSK, Vifor Pharmaceuticals, AstraZeneca and Respiri UK, outside the submitted work.

\section{References}

1 Grigg J, Barben J, Everard ML, et al. Key paediatric messages from the 2017 European Respiratory Society International Congress. ERJ Open Res 2018; 4: 00165-2017.

2 Grigg J, Balfour-Lynn IM, Everard M, et al. Key paediatric messages from the 2016 European Respiratory Society International Congress. ERJ Open Res 2017; 3: 00127-2016.

3 Bizzotto D, Dahl M, Veneroni C, et al. Effects of sustained lung inflation (SLI) at birth on lung aeration during non-invasive resuscitation of preterm lambs. Eur Respir J 2018; 52: Suppl. 62, OA304.

4 Foglia EE, Owen LS, Thio M, et al. Sustained Aeration of Infant Lungs (SAIL) trial: study protocol for a randomized controlled trial. Trials 2015; 16: 95.

5 Bruschettini M, O'Donnell CPF, Davis PG, et al. Sustained versus standard inflations during neonatal resuscitation to prevent mortality and improve respiratory outcomes. Cochrane Database Syst Rev 2017; 7: CD004953.

6 Dahl MJ, Veneroni C, Lavizzari A, et al. Lung mechanics, airway reactivity, and muscularization are altered in former mechanically ventilated preterm lambs. Eur Respir J 2018; 52: Suppl. 62, OA303.

7 Sampath V, Garland JS, Helbling D, et al. Antioxidant response genes sequence variants and BPD susceptibility in VLBW infants. Pediatr Res 2015; 77: 477-483.

8 Pavlinova E, Savchenko O, Vlasenko N, et al. The analysis of genetic polymorphisms of manganese superoxide dismutase at premature infants with bronchopulmonary dysplasia. Eur Respir J 2018; 52: Suppl. 62, OA307.

9 Zannin E, Dellacà R, Neumann R, et al. Assessment of lung mechanics for the prediction and evaluation of pulmonary outcome in preterm infants. Eur Respir J 2018; 52: Suppl. 62, OA306.

10 Kindt-Dunjko A, Foerster K, Flemmer A, et al. Protein signature stratifies severity of chronic lung disease in preterms after birth. Eur Respir J 2018; 52: Suppl. 62, OA300.

11 Gallacher D, Triantafilou M, Marchesi J, et al. The role of ASC signalling in preterm infants at risk of chronic lung disease of prematurity. Eur Respir J 2018; 52: Suppl. 62, OA302.

12 Nair V, Loganathan P, Soraisham AS. Azithromycin and other macrolides for prevention of bronchopulmonary dysplasia: a systematic review and meta-analysis. Neonatology 2014; 106: 337-347. 

dysplasia in preterm infants: a randomized, double-blind, placebo-controlled trial. Pediatr Pulmonol 2011; 46: $111-118$.

14 Viscardi R, Terrin M, Magder L, et al. Azithromycin (AZM) reduces death or severe respiratory morbidity at 12 month adjusted age (AA) in urea plasma-positive intubated preterms. Eur Respir J 2018; 52: Suppl. 62, OA301.

15 Harris C, Lunt A, Peacock J, et al. Are lung function abnormalities associated with reduced exercise capacity in prematurely born young people? Eur Respir J 2018; 52: Suppl. 62, OA305.

16 Danopoulos S, Bhattacharya S, Thornton M, et al. Diversity in mesenchymal lineages during early human lung development. Eur Respir J 2018; 52: Suppl. 62, PA1378.

17 Wang K, Elliot J, Saglani S, et al. Thickening of the airway smooth muscle layer from late gestation to first year of life is accompanied by a reduction in smooth muscle cell density. Eur Respir J 2018; 52: Suppl. 62, PA1388.

18 Miura A, Yanagi S, Tsubouchi H, et al. The role of epithelial Pten in programmed cellular senescence during lung development. Eur Respir J 2018; 52: Suppl. 62, PA1386.

19 Collins J, Lithopoulos MA, Dos Santos CC, et al. Impaired angiogenic supportive capacity and altered gene expression profile of CD146+ mesenchymal stromal cells isolated from hyperoxia-injured neonatal rat lungs. Eur Respir J 2018; 52: Suppl. 62, PA1377.

20 Collins JJP, Lithopoulos MA, Dos Santos CC, et al. Impaired angiogenic supportive capacity and altered gene expression profile of resident CD146(+) mesenchymal stromal cells isolated from hyperoxia-injured neonatal rat lungs. Stem Cells Dev 2018; 27: 1109-1124.

21 Hirani D, Dinger K, Mohr J, et al. Loss of IL-6 enables lung growth in newborn mice exposed to prolonged hyperoxia. Eur Respir J 2018; 52: Suppl. 62, A1381.

22 Hirani D, Koch M, Dinger K, et al. Krüppel-like factor 4 (Klf4) is a novel regulator of neonatal lung fibroblast homeostasis and reduced in hyperoxia-induced lung injury. Eur Respir J 2018; 52: Suppl. 62, PA1379.

23 Mohr J, Koch M, Oikonomou N, et al. Krüppel-like Factor 4 (Klf4) in human and mouse lung development: regulation of ATII cell homeostasis in lungs of newborn mice exposed to HYX. Eur Respir J 2018; 52: Suppl. 62, PA1385.

24 Syed MA, Shah D, Bhandari V. Role of TREM1-Ripk3 axis in hyperoxia induced lung injury in neonates. Eur Respir J 2018; 52: Suppl. 62, PA1382.

25 Katovich I, Rutkouskaya Z, Anischenko S, et al. Effect of inhaled N-acetylcysteine on the oxidative stress and content of proteases in lungs of newborn guinea pigs exposed to hyperoxia. Eur Respir J 2018; 52: Suppl. 62, PA1387.

26 Ljujich M, Varga M, Bartel S, et al. Zebrafish as a model for study of developmental origins of chronic lung diseases. Eur Respir J 2018; 52: Suppl. 62, PA1389.

27 Pincus A, Fryer A, Jacoby D, et al. Maternal allergen exposure increases offspring sensory airway innervation and airway hyperreactivity in mice. Eur Respir J 2018; 52: Suppl. 62, PA1380.

28 Kovach M, Käck U, Che K, et al. Systemic IL-26 correlates with improved asthma control in children with allergic sensitization. Eur Respir J 2018; 52: Suppl. 62, PA1376.

29 van de Loo KF, van Gelder MM, Roukema J, et al. Prenatal maternal psychological stress and childhood asthma and wheezing: a meta-analysis. Eur Respir J 2016; 47: 133-146.

30 Liu X, Madsen K, Sejbæk C, et al. Prenatal exposure to negative life events and job stress and asthma risk Eur Respir J 2018; 52: Suppl. 62, OA335.

31 Hämynen I, Täubel M, Karvonen A, et al. Breast-milk microbes and risk of asthma by 6 years of age. Eur Respir J 2018; 52: Suppl. 62, OA336.

32 Ege MJ, Mayer M, Normand AC, et al. Exposure to environmental microorganisms and childhood asthma. N Engl J Med 2011; 364: 701-709.

33 Pekkanen J, Karvonen A, Adams R, et al. Microbiota as in farm homes protect children from asthma. Eur Respir J 2018; 52: Suppl. 62, OA337.

34 Brand PL, Baraldi E, Bisgaard H, et al. Definition, assessment and treatment of wheezing disorders in preschoo children: an evidence-based approach. Eur Respir J 2008; 32: 1096-1110.

35 Townshend J, Hails S, McKean M. Diagnosis of asthma in children. BMJ 2007; 335: 198-202.

36 Eckel S, Zhang Y, Garcia E, et al. Age and height trajectories of exhaled nitric oxide in schoolchildren. Eur Respir J 2018; 52: Suppl. 62, OA339.

37 Fens N, Zwinderman AH, van der Schee MP, et al. Exhaled breath profiling enables discrimination of chronic obstructive pulmonary disease and asthma. Am J Respir Crit Care Med 2009; 180: 1076-1082.

38 de Heer K, van der Schee MP, Zwinderman K, et al. Electronic nose technology for detection of invasive pulmonary aspergillosis in prolonged chemotherapy-induced neutropenia: a proof-of-principle study. J Clin Microbiol 2013; 51: 1490-1495.

39 Bannier M, van de Kant KDG, Jobsis Q, et al. Feasibility and diagnostic accuracy of an electronic nose in children with asthma and cystic fibrosis. J Breath Res 2018; https://doi.org/10.1088/1752-7163/aae158.

40 Csonka P, Lehtimaki L. Valved holding chambers vary considerably in drug delivery efficacy. Eur Respir J 2018 52: Suppl. 62, OA340.

41 Compton V, Peak M, Grice J, et al. Humidified and standard oxygen therapy in acute severe asthma in children a randomised controlled pilot trial (HUMOX). Eur Respir J 2018; 52: Suppl. 62, OA341.

42 Magnus MC, Karlstad O, Haberg SE, et al. Prenatal and infant paracetamol exposure and development of asthma: the Norwegian Mother and Child Cohort Study. Int J Epidemiol 2016; 45: 512-522.

43 Kallen B, Finnstrom O, Nygren KG, et al. Maternal drug use during pregnancy and asthma risk among children. Pediatr Allergy Immunol 2013; 24: 28-32.

44 Brew B, Almqvist C, Lundholn C, et al. Prescribed analgesics in pregnancy and the risk of child asthma - a register-based study. Eur Respir J 2018; 52: Suppl. 62, OA3305.

45 Ortqvist AK, Lundholm C, Wettermark B, et al. Validation of asthma and eczema in population-based Swedish drug and patient registers. Pharmacoepidemiol Drug Saf 2013; 22: 850-860.

46 Dai X, Dharmage S, Abramson M, et al. GST genotypes modify the association between paracetamol use in early life and lung function at 18 years. Eur Respir J 2018; 52: Suppl. 62, OA3309. 
Lowe AJ, Lodge CJ, Allen KJ, et al. Cohort profile: Melbourne Atopy Cohort study (MACS). Int J Epidemio 2017; 46: 25-26.

Kotecha S, Watkins J, Lowe J, et al. Association of early life factors with wheezing phenotypes in preterm-born children compared to term-born children. Eur Respir J 2018; 52: Suppl. 62, OA3306.

Connelly R, Platt L. Cohort profile: UK Millennium Cohort Study (MCS). Int J Epidemiol 2014; 43: 1719-1725.

Sanahuja M C, Dekker H D, Kruithof C, et al. Early childhood growth patterns and lung function and asthma at 10 years. Eur Respir J 2018; 52: Suppl. 62, OA3308.

Claudia F, Thiering E, von Berg A, et al. Peak weight velocity in infancy is negatively associated with lung function in adolescence. Pediatr Pulmonol 2016; 51: 147-156.

Sonnenschein-van der Voort AM, Howe LD, Granell R, et al. Influence of childhood growth on asthma and lung function in adolescence. J Allergy Clin Immunol 2015; 135: 1435-1443.

Jaddoe VW, van Duijn CM, Franco OH, et al. The Generation R Study: design and cohort update 2012. Eur J Epidemiol 2012; 27: 739-756.

Kho AT, Sharma S, Qiu W, et al. Vitamin D related genes in lung development and asthma pathogenesis. BMC Med Genomics 2013; 6: 47.

Song H, Yang L, Jia C. Maternal vitamin D status during pregnancy and risk of childhood asthma: a meta-analysis of prospective studies. Mol Nutr Food Res 2017; 61.

Mensink-Bout R, Van Meel E, De Jongste JC, et al. Early life vitamin D levels and childhood respiratory morbidity and allergic sensitization. Eur Respir J 2018; 52: Suppl. 62, OA3307.

An SQ, Warris A, Turner S. Microbiome characteristics of induced sputum compared to bronchial fluid and upper airway samples. Pediatr Pulmonol 2018; 53: 921-928.

Poh MWP, Fonceca A, Kicic A, et al. Assessing polymicrobial interactions in a 3D primary airway epithelial cell model. Eur Respir J 2018; 52: Suppl. 62, OA3603.

Pierangeli A, Scagnolari C, Bitossi C, et al. Interferon-stimulated gene expression differs in bronchiolitis caused by RSV-A strains NA1 and ON1. Eur Respir J 2018; 52: Suppl. 62, OA3604.

Cangiano G, Guarise R, Borruso A, et al. 'Effects' of bronchiolitis on ventilation inhomogeneity (VI) at preschool age: comparison of multiple breath washout (MBW) between post-bronchiolitis, cystic fibrosis and healthy children. Eur Respir J 2018; 52: Suppl. 62, OA3606.

Riikonen R, Lauhkonen E, Törmänen S, et al. Infant bronchiolitis increases the risk of reduced lung function at age 10-13 years. Eur Respir J 2018; 52: Suppl. 62, OA3607.

Riikonen R, Lauhkonen E, Tormanen S, et al. Prospective study confirms that bronchiolitis in early infancy increases the risk of reduced lung function at 10-13 years of age. Acta Paediatr 2019; 108: 124-130.

Unger S, Halliday C, Ziaie A, et al. Blood gas analysis as a marker of severity in acute bronchiolitis. Eur Respir 2018; 52: Suppl. 62, OA3605.

Ruffles T, Marchant J, Masters I, et al. Outcomes in protracted bacterial bronchitis (PBB): a five year prospective cohort study. Eur Respir J 2018; 52: Suppl. 62, OA3608.

Elborn J. Cystic fibrosis. Lancet 2016; 388: 2519-2531.

Linnane B, Cotter P, O'Sullivan O, et al. Prospective case-control study describing the lower airway microbiome in pre-school children with cystic fibrosis. Eur Respir J 2018; 52: Suppl. 62, OA509.

Neerincx AH, Brinkman P, van der Gouwe P, et al. Detection of Pseudomonas aeruginosa in exhaled breath of cystic fibrosis patients. Eur Respir J 2018; 52: Suppl. 62, OA511.

Baghdasaryan A, Bruderer T, Mueller S, et al. Distinct volatile markers from cystic fibrosis pathogens with secondary electrospray ionisation high-resolution mass spectrometry (SESI-HRMS). Eur Respir J 2018; 52: Suppl. 62, OA512.

Stahl M, Wielpütz MO, Ricklefs I, et al. Preventive inhalation of hypertonic saline in infants with cystic Fibrosis (PRESIS): a randomized, double-blind, controlled study. Am J Respir Crit Care Med 2018; https://doi.org/10 1164/rccm.201807-1203OC. treatment of cystic fibrosis. Eur Respir J 2018; 52: Suppl. 62, OA514.

McDonald R, Stanojevic S, Jensen R, et al. Do deviations in tidal breathing impact multiple breath washout measurements? Eur Respir J 2018; 52: Suppl. 62, OA4987.

Ciuca IM, Dediu M, Pop LL. Lung clearance index and lung ultrasound in cystic fibrosis children. Eur Respir J 2018; 52: Suppl. 62, OA4988.

Nyilas S, Bauman G, Pusterla O, et al. Ventilation and perfusion assessed by functional MRI in children with CF: reproducibility in comparison to lung function. J Cyst Fibros 2018; https://doi.org/10.1016/j.jcf.2018.10.003.

Smith LJ, Collier GJ, Marshall H, et al. Patterns of regional lung physiology in cystic fibrosis using ventilation MRI and MBW. Eur Respir J 2018; 52: 1800821.

Hardy S, Berardis S, Reychler G, et al. Comparison between the 1-minute sit-to-stand test and quadriceps strength in patients with cystic fibrosis. Eur Respir J 2018; 52: Suppl. 62, OA4991.

Frost F, Dyce P, Wat D, et al. Glycaemic variability indices are associated with increased pulmonary function decline in people with cystic fibrosis. Eur Respir J 2018; 52: Suppl. 62, OA4992.

Shoemark A, Lucas JS. Diagnosis of primary ciliary dyskinesia: current practices and future prospective. In: Chalmers JD, Polverino E, Aliberti S, eds. Bronchiectasis. Sheffield, European Respiratory Monograph 2018 81: 267-281.

Kuehni CE, Goutaki M, Rubbo B, et al. Management of primary ciliary dyskinesia: current practices and future prospectives. In: Chalmers JD, Polverino E, Aliberti S, eds. Bronchiectasis. Sheffield, European Respiratory Monograph 2018; 81: 282-299.

Behan L, Dimitrov BD, Kuehni CE, et al. PICADAR: a diagnostic predictive tool for primary ciliary dyskinesia. Eur Respir J 2016; 47: 1103-1112. in children and adolescents. Ann Am Thorac Soc 2016; 13: 1305-1313.

unique clinical and ciliary phenotype. Am J Respir Crit Care Med 2014; 189: 707-717. 

ciliary dyskinesia disrupts protein oligomerisation and is associated with normal diagnostic investigations. Thorax 2018; 73: 157-166.

83 Marthin JK, Philipsen MC, Rosthoj S, et al. Infant nasal NO over time; natural evolution and impact of respiratory tract infection. Eur Respir J 2018; 51: 1702503.

84 Lucas JS, Evans HJ, Haarman EG, et al. Exploring the Art of Ciliary Beating. Chest 2017; 152: 1348-1349.

85 Omran H, Kobayashi D, Olbrich H, et al. KTU/PF13 is required for cytoplasmic pre-assembly of axonemal dyneins. Nature 2008; 456: 611-616.

86 Paff T, Loges NT, Aprea I, et al. Mutations in PIH1D3 cause X-linked primary ciliary dyskinesia with outer and inner dynein arm defects. Am J Hum Genet 2017; 100: 160-168.

87 Davis SD, Rosenfeld M, Lee HS, et al. Primary ciliary dyskinesia: longitudinal study of lung disease by ultrastructure defect and genotype. Am J Respir Crit Care Med 2019; 199: 190-198.

88 Lucas JS, Barbato A, Collins SA, et al. European Respiratory Society guidelines for the diagnosis of primary ciliary dyskinesia. Eur Respir J 2017; 49: 1601090.

89 Shapiro A, Davis SD, Polineni D, et al. Diagnosis of primary ciliary dyskinesia. An official American Thoracic Society clinical practice guideline. Am J Respir Crit Care Med 2018; 197: e24-e39.

90 Shah A, Shoemark A, MacNeill SJ, et al. A longitudinal study characterising a large adult primary ciliary dyskinesia population. Eur Respir J 2016; 48: 441-450.

91 Halbeisen F, Goutaki M, Spycher BD, et al. Lung function in patients with primary ciliary dyskinesia: an iPCD Cohort study. Eur Repir J 2018; 52: 1801040.

92 Vanaken GJ, Bassinet L, Boon $\mathrm{M}$, et al. Infertility in an adult cohort with primary ciliary dyskinesia: phenotype-gene association. Eur Respir J 2017; 50: 1700314.

93 Barbato A, Frischer T, Kuehni CE, et al. Primary ciliary dyskinesia: a consensus statement on diagnostic and treatment approaches in children. Eur Respir J 2009; 34: 1264-1276.

94 Shapiro AJ, Zariwala MA, Ferkol T, et al. Diagnosis, monitoring, and treatment of primary ciliary dyskinesia: PCD foundation consensus recommendations based on state of the art review. Pediatric Pulmonol 2016; 51: $115-132$.

95 Paff T, Daniels JM, Weersink EJ, et al. A randomised controlled trial on the effect of inhaled hypertonic saline on quality of life in primary ciliary dyskinesia. Eur Respir J 2017; 49: 1601770.

96 Kobbernagel HE, Buchvald FF, Haarman EG, et al. Study protocol, rationale and recruitment in a European multi-centre randomized controlled trial to determine the efficacy and safety of azithromycin maintenance therapy for 6 months in primary ciliary dyskinesia. BMC Pulm Med 2016; 16: 104.

97 Weber S, Castro Correa C, Evangelisti M, et al. Sensibility and specificity of the sleep clinical record protocol in Brazilian children. Eur Respir J 2018; 52: Suppl. 62, OA3585.

98 Evangelisti M, Barreto M, Cecili M, et al. Lingual frenulum evaluation in school age children with and without sleep disordered breathing. Eur Respir J 2018; 52: Suppl. 62, OA3588.

99 Truccio F, Bush A, Tan HL. Role of $\mathrm{CO}_{2}$ monitoring in the diagnosis and management of paediatric sleep disordered breathing. Eur Respir J 2018; 52: Suppl. 62, OA3592.

100 Wright $\mathrm{M}$, Javadpour S, Jackson $\mathrm{P}$, et al. Management of upper airway obstruction in infants with Robin sequence in the United Kingdom and Republic of Ireland. Eur Respir J 2018; 52: Suppl. 62, OA3589.

101 Owens L, Laing I, Murdzoska J, et al. Glutathione s-transferase genotype determines lung function deficits from infancy to adulthood in those with in utero tobacco exposure. Eur Respir J 2018; 52: Suppl. 62, OA3586.

102 Beydon N, Dufetelle E, Bokov P, et al. Should bronchodilator response be assessed in all asthmatic children with normal baseline spirometry. Eur Respir J 2018; 52: Suppl. 62, OA3587.

103 Verheggen M, Okitika T, Shackleton C, et al. Validation of the hypoxia challenge test for preterm infants. Eur Respir J 2018; 52: Suppl. 62, OA3590.

104 Usemann J, Suter A, Zannin E, et al. Variability of tidal breathing parameters in preterm infants and associations with respiratory morbidity during infancy: a cohort Study. J Pediatr 2019; 205: 61-69. 